\title{
Origin, diversification, and historical biogeography of the giant kelps genus Macrocystis: Evidences from Bayesian phylogenetic analysis
}

\author{
Origen, diversificación y biogeografía histórica de macroalgas del género Macrocystis: \\ Evidencia desde análisis filogenéticos bayesianos

\section{Marcela P. Astorga ${ }^{1}$, Cristián E. Hernández ${ }^{2}$, Cynthia P. Valenzuela ${ }^{1}$, Jorge Avaria-Llautureo ${ }^{2,3}$ and Renato Westermeier ${ }^{1}$}

\begin{abstract}
${ }^{1}$ Instituto de Acuicultura, Universidad Austral de Chile, Sede Puerto Montt, Casilla 1327, Puerto Montt, Chile. marcelaastorga@spm.uach.cl

${ }^{2}$ Laboratorio de Ecología Molecular y Filoinformática, Departamento de Zoología, Facultad de Ciencias Naturales y Oceanográficas, Universidad de Concepción, Casilla 160-C, Concepción, Chile

${ }^{3}$ Programa de Magíster en Ciencias Mención Zoología, Departamento de Zoología, Facultad de Ciencias Naturales y Oceanográficas, Universidad de Concepción, Casilla 160-C, Concepción, Chile
\end{abstract}

\begin{abstract}
We evaluate the genetic divergence between the two morphs of the genus Macrocystis found in the Chilean coast (pyrifera and integrifolia), and the historical relationships with the other morphs of the genus using the ITS-2 DNA region sequences. The analyses sustain that the morphotypes, and its distribution, have a strong phylogenetic signal, indicating that historical process associated to the diversification has determined the current distribution and morphological variability. Finally, the pyrifera morph is the most probable ancestor of the actual morphological variability of the genus, which originated in the Northeastern Pacific and later colonized the Southern Pacific.
\end{abstract}

Key words: Bayesian phylogenetics, Comparative method, ITS

\section{INTRODUCTION}

The giant kelps, genus Macrocystis, are large brown algae of ecological importance due to the forests that they form in the temperate regions of both hemispheres (Graham et al. 2007). The classification of the number of species belonging to this genus has been the subject of much discussion, due to the high morphological variability in the characters utilized. A high diversity of forms has often been referred to as ecotypes (Graham et al. 2007, Demes et al. 2009). The most utilized classification includes 4 species (i.e., M. pyrifera, $M$. integrifolia, $M$. angustifolia and $M$. laevis), however the genus has recently been synonymized into a single species (i.e., M. pyrifera) (Demes et al. 2009, Macaya \& Zuccarello 2010). This reorganization of the genus is concordant with the production of fertile offspring between morphospecies (Lewis et al. 1986, Druehl et al. 2005, Westermeier et al. 2007); with no support for the separation of the genus into its 4 species based on a molecular phylogeny (Coyer et al. 2001); with high plasticity in kelp blade morphology induced by environmental factors (Demes et al. 2009); and with low genetic variation in the 4 ecomorphs (Macaya \& Zuccarello 2010).
The 4 most commonly identified morphs of Macrocystis show different ranges of distribution: pyrifera and integrifolia are present in both hemispheres, while laevis and angustifolia are present only in the Southern Hemisphere. Biogeographic studies of the genus Macrocystis suggest that the current global antitropical distribution originated with the emergence of the genus in the Northern Hemisphere and a later colonization of the Southern Hemisphere (Lindberg 1991). Alternatively, Chin et al. (1991) proposed an origin in the Southern Hemisphere, however, this hypothesis has been questioned (Lindberg 1991).

Specifically, Coyer et al. (2001) described the phylogeography of the genus using ITS region, suggesting a recent dispersal of Macrocystis from the Northern Hemisphere to the Southern Hemisphere and a crossing of the tropical barrier between 3.1 mya to 10,000 years ago, based on the paraphyletic relationship of northern hemisphere individuals relative to the wellsupported monophyletic origin of Southern Hemisphere individuals, and the greater sequence diversity within 
the Northern Hemisphere individuals. Additionally, Macaya \& Zucarrello (2010) sampled 7 localities along the South-eastern Pacific coast and performed molecular studies of COI sequences, using the morph integrifolia is present along the Peruvian and northern Chilean coast $\left(6-32^{\circ} \mathrm{S}\right)$, and the morph pyrifera is distributed from $37^{\circ} \mathrm{S}$ down to Patagonia (55º) (Dayton 1985).

Although the origin of the giant kelp has been already suggested by Nicholson (1978), Estes \& Steinberg (1988) and Lüning (1990), among others, we want to add information to this discussion that may allow a contribution to answer a series of questions about the origin and diversification of this genus: 1.- Did the genus originate in the northern hemisphere, or is the origin more likely to have been in the southern hemisphere as proposed by Chin et al. (1991)?, 2.- Specifically, which ocean could the common ancestor of the genus most likely inhabited?, 3.- Given the large diversity of morphotypes, which morphotype is most likely the ancestral form of this genus?

The present study evaluates the 3 questions outlined above through the use of Bayesian phylogenetic approximations and the phylogenetic comparative method. It also aims to generate a more complete picture concerning the origin and diversification of the genus Macrocystis, through the utilization of sequences of the ITS-2 region. Moreover we determine the genetic divergence between the two morph species in Chile and evaluate the hypothesis of Coyer et al. (2001) concerning the origin and diversification of the genus Macrocystis.

\section{Materials ANd Methods}

On the coast of Chile, individuals of the integrifolia morph were sampled from Playa Blanca ( $26^{\circ} 20^{\prime} \mathrm{S}$; $70^{\circ} 36^{\prime} \mathrm{W}$ ), and individuals of pyrifera were collected from Teupa

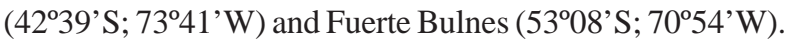

The extraction of DNA from the samples was done using the method of phenol/chloroform/isoamyl alcohol, with precipitation in ethanol (Doyle \& Doyle 1987). An amplification of DNA from the ITS-2 region was done initially utilizing the primers and protocols described by Druehl et al. (2005). Based on the first sequences, a new reverse primer was designed for which the program Webprimer ${ }^{1}$ was utilized. The evaluation of the designed oligonucleotide was done using the software Net Primer². The new oligo sequence was ITSR: 5'- ctc ttg ctt gat cgg agg tc- 3 '. The sequences were edited and aligned utilizing the programs BIOEDIT 5.0.9 (Hall 1999).

The sequences obtained were analyzed including sequences available in GenBank for all known distribution areas (Coyer et al. 2001, Yoon et al. 2001, Lane et al. 2006) of the other morphs: pyrifera and integrifolia from the Pacific coasts of Canada and the United States, laevis from Marion Island (South Africa), and angustifolia from New Zealand and Australia. The species Pelagophycus porra was utilized as outgroup. The genetic divergence between samples was estimated through to the number of nucleotide differences between groups $(\mathrm{k})$, using Kimura 2-parameters model, and the average number of nucleotide substitutions within and between samples $\left(D_{x y}\right)$. These statistics were calculated using DnaSP version 5.00.04 (Librado \& Rozas 2009).

To estimate the phylogenetic relationships a general likelihood-based mixture model (MM) of gene-sequence evolution was applied (Pagel \& Meade 2004). The MM model, implemented in a Bayesian Markov Chain Monte Carlo (BMCMC) framework, was used to estimate the posterior probability of the phylogenetic trees to include this information in the comparative analyses. The Reversible-Jump Markov Chain Monte Carlo (RJMCMC) procedure (Pagel \& Meade 2006) was used to find the best MM that summarizes the sequence evolution using BayesPhylogenies 1.1 software ${ }^{3}$. The BMCMC analysis was run using 129,930,000 generations of phylogenetic trees, sampling every $10,000^{\text {th }}$. From the sample of trees, the first 100 of the sample were removed to avoid the inclusion of trees sampled before the convergence of the Markov Chain, and we re-sampled every 11 trees to obtain a final sample of 1173 independent trees.

The phylogenetic signal of Macrocystis traits (i.e., ocean distribution and morphotype) was evaluated using the association index statistic (AI), and its significance incorporated phylogenetic uncertainty as follows: First, the value of AI was calculated for every tree in the posterior sample of trees, forming the posterior distribution of the statistic with a observed median $\mu$; next, from the observed traits-set for the individuals $C$, we generated 100 randomized traits-sets. Then, for each $\mathrm{C}_{\mathrm{i}}$ the median posterior estimate of the statistic $\left(\mu_{\mathrm{i}}\right)$ was

\footnotetext{
${ }^{1}<$ http://genome-www2.stanford.edu/cgi-bin/SGD/web-primer>

${ }^{2}<$ http://www.premierbiosoft.com/netprimer/netprlaunch/netprlaunch.html>

${ }^{3}<$ http://www. evolution.rdg.ac.uk/BayesPhy.html>
} 
calculated from the posterior sample of trees. Finally, the $\mathrm{p}$-value was then obtained from this null distribution by calculating the proportion of $\mu_{\mathrm{i}}$ values that are more extremes than the observed value $\mu$. This analysis was conducted using BaTS software ${ }^{4}$ (Parker et al. 2008).

To reconstruct the ancestral states the MCMC method was used (Pagel et al. 2004) with the continuous-time Markov k-state model (Lewis 2001) implemented in BayesTraits software (Pagel \& Meade 2009). States were identified as discrete for distribution (i.e., (0) Northern Hemisphere vs. (1) Southern Hemisphere; (0) Northeast Pacific Ocean, (1) Southeast Pacific Ocean, (2) Southern Atlantic Ocean, (3) Indian Ocean, and (4) Southwest Pacific Ocean), and for morphologies, i.e., (p) pyrifera, (i) integrifolia, (a) angustifolia, and (l) laevis of the Macrocystis genus. To estimate the most probable ancestral state of characters we used the consensus phylogenetic tree with both the topology and mean branch lengths obtained from the BMCMC molecular phylogenetic analysis. With these analyses we were able to relate the evolutionary event of the origin of Macrocystis, with the estimated distribution and morphology of the ancestral states.

\section{Results And Discussion}

We obtained a final aligned DNA-matrix of ITS-2 region for 53 taxa (15 of this study; 38 of GenBank), with an extension of $396 \mathrm{pb}$. The genetic divergence between both morphs in the Chilean coast measured by average number of nucleotides substitution per site was $\mathrm{D} x_{y}=0.013 \pm$ 0.006 , and the average number of nucleotide differences between groups was $\mathrm{k}=4.917$. The genetic divergence within each group using Kimura 2-parameters was $d=$ $0.001 \pm 0.001$ for pyrifera group and $\mathrm{d}=0.004 \pm 0.002$ for integrifolia group. The genetic divergence among the same morph but in different hemisphere was $\mathrm{D} x_{y}=0.019 \pm$ 0.005 and $\mathrm{k}=8.724 \pm 1.502$ for pyrifera group and $\mathrm{D} x_{y}=$ $0.032 \pm 0.007$ and $\mathrm{k}=10.333 \pm 1.905$ for integrifolia group.

The phylogenetic reconstruction that best adjusts to the rates and patterns of the molecular evolution was the one obtained with $1 \mathrm{GTR}+\Gamma$ matrix. The phylogeny showed a separation between pyrifera and integrifolia morphs from the Chilean coast, however, on a global level, consistency in the separation between these morphs was not observed (Fig. 1). In fact, high values of divergence between samples of the same morph between different hemispheres were observed (Fig. 1).

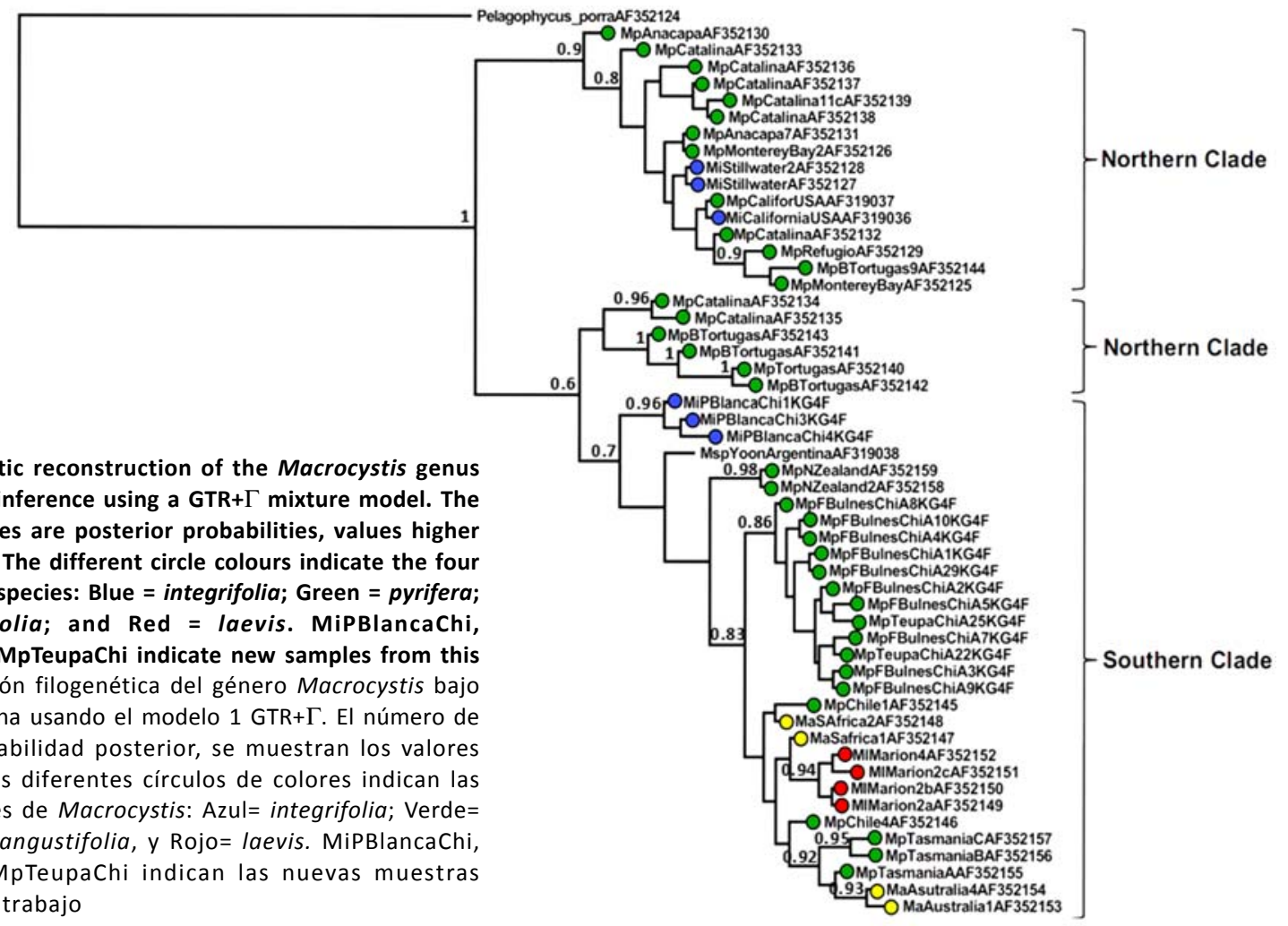

Figure 1. Phylogenetic reconstruction of the Macrocystis genus under the Bayesian inference using a GTR $+\Gamma$ mixture model. The numbers at the nodes are posterior probabilities, values higher than $\mathbf{0 . 5}$ are shown. The different circle colours indicate the four Macrocystis morphospecies: Blue = integrifolia; Green = pyrifera; Yellow = angustifolia; and Red = laevis. MiPBlancaChi, MpFBulnesChi, and MpTeupaChi indicate new samples from this work / Reconstrucción filogenética del género Macrocystis bajo la inferencia Bayesiana usando el modelo $1 \mathrm{GTR}+\Gamma$. El número de los nodos son probabilidad posterior, se muestran los valores superiores a 0,5. Los diferentes círculos de colores indican las cuatro morfoespecies de Macrocystis: Azul= integrifolia; Verde= pyrifera; Amarillo= angustifolia, y Rojo= laevis. MiPBlancaChi, MpFBulnesChi, y MpTeupaChi indican las nuevas muestras aportadas por este trabajo

${ }^{4}$ Bayesian Tip-association Significance testing. <http://evolve.zps.ox.ac.uk/evolve/BaTS.html> 
These results support the proposal of Coyer et al. (2001), and based on the results achieved through the utilization of Bayesian phylogenetic methods, we support the hypothesis that the morphospecies belonging to the genus Macrocystis are in fact a single species, M. pyrifera, like was previously proposed by Demes et al. (2009) and by Macaya \& Zucarrello (2010). Our results suggest that this genus is actually in the process of speciation through geographic isolation or a recent breakage in genetic flow, in which genetic similarity is dominated by the geographic proximity, but the phenotypic similarity is dominated by different habitats (Demes et al. 2009), that have been referred to as ecotype (Graham et al. 2007). The incipient genetic differentiation between the integrifolia and pyrifera morphs from South America is potentially explained because they inhabit different environments. The morphs of Macrocystis found on the Chilean coast do not form differentiated clades, and the genetic flow is still occurring between both groups, a process that has

A) Hemisphere

\begin{tabular}{ccc}
\hline Node & \multicolumn{2}{c}{ Ancestral State } \\
\cline { 2 - 3 } & Northern Hemisphere & Southern Hemisphere \\
\hline 1 & $0.88 \pm 0.13$ & $0.12 \pm 0.13$ \\
2 & $0.99 \pm 0.01$ & $0.01 \pm 0.01$ \\
3 & $0.64 \pm 0.22$ & $0.36 \pm 0.22$ \\
4 & $0.99 \pm 0.01$ & $0.01 \pm 0.01$ \\
5 & $0.01 \pm 0.01$ & $0.99 \pm 0.01$ \\
\hline
\end{tabular}

been demonstrated in the laboratory (Lewis et al. 1986, Westermeier et al. 2007).

Results show that phylogenetically related individuals are more similar than expected by chance in their distribution by Hemisphere (Observed AI $=0<$ Null AI mean $=2.68, P<0.0001)$, Ocean $($ Observed $\mathrm{AI}=0.15<$ Null AI mean $=3.92, P<0.0001)$, and morphology $($ Observed AI $=1.41<$ Null AI mean $=2.61, P<0.0001)$. (Table 1). The BMCMC method showed that the ancestor of the Macrocystis genus had a high probability of inhabiting the Northern Hemisphere (Fig. 2A, Table 1A) on the Northeastern Pacific coast (Fig. 2B, Table 1B). The only clade that had a high probability of an ancestor from Southern Hemisphere was the Southern Clade (Fig. 2A, Table 1A), with a common ancestor from the Southeastern Pacific coast (Fig. 2B, Table 1B). We found that all of the individuals of the Macrocystis genus had a high probability of descending from a pyrifera morph ancestor (Fig. 2C, Table 1C).

Table 1. The probability values for each ancestral state of (A) Hemisphere, (B) Ocean, and (C) Morphology of Macrocystis. The probability values are reported with 10 times the standard error based on the BMCMC approach / Valores de probabilidad de cada estado ancestral para (A) Hemisferio, (B) Océano y (C) Morfología de Macrocystis. Los valores de probabilidad son reportados con 10 veces el error estándar basado en la aproximación BMCMC

B) Ocean

\begin{tabular}{cccccc}
\hline Node & \multicolumn{5}{c}{ Ancestral State } \\
\cline { 2 - 5 } & Northeast Pacific & Southeast Pacific & South Atlantic & South Indian & Southwest Pacific \\
\hline 1 & $0.38 \pm 0.11$ & $0.13 \pm 0.04$ & $0.17 \pm 0.03$ & $0.17 \pm 0.03$ & $0.15 \pm 0.04$ \\
2 & $0.84 \pm 0.08$ & $0.02 \pm 0.01$ & $0.06 \pm 0.04$ & $0.06 \pm 0.04$ & $0.04 \pm 0.03$ \\
3 & $0.29 \pm 0.10$ & $0.16 \pm 0.06$ & $0.22 \pm 0.06$ & $0.19 \pm 0.06$ & $0.14 \pm 0.05$ \\
4 & $0.76 \pm 0.11$ & $0.03 \pm 0.02$ & $0.08 \pm 0.05$ & $0.08 \pm 0.05$ & $0.05 \pm 0.04$ \\
5 & $0.02 \pm 0.01$ & $0.41 \pm 0.11$ & $0.23 \pm 0.06$ & $0.18 \pm 0.06$ & $0.16 \pm 0.06$ \\
\hline
\end{tabular}

C) Morphology

\begin{tabular}{ccccc}
\hline \multirow{2}{*}{ Node } & \multicolumn{4}{c}{ Ancestral State } \\
\cline { 2 - 5 } & pyrifera & integrifolia & angustifolia & laevis \\
\hline 1 & $0.30 \pm 0.05$ & $0.23 \pm 0.02$ & $0.24 \pm 0.02$ & $0.22 \pm 0.02$ \\
2 & $0.64 \pm 0.10$ & $0.13 \pm 0.06$ & $0.13 \pm 0.05$ & $0.11 \pm 0.05$ \\
3 & $0.31 \pm 0.08$ & $0.27 \pm 0.05$ & $0.22 \pm 0.04$ & $0.20 \pm 0.05$ \\
4 & $0.56 \pm 0.10$ & $0.15 \pm 0.05$ & $0.16 \pm 0.05$ & $0.13 \pm 0.05$ \\
5 & $0.12 \pm 0.04$ & $0.44 \pm 0.08$ & $0.23 \pm 0.05$ & $0.21 \pm 0.06$ \\
\hline
\end{tabular}




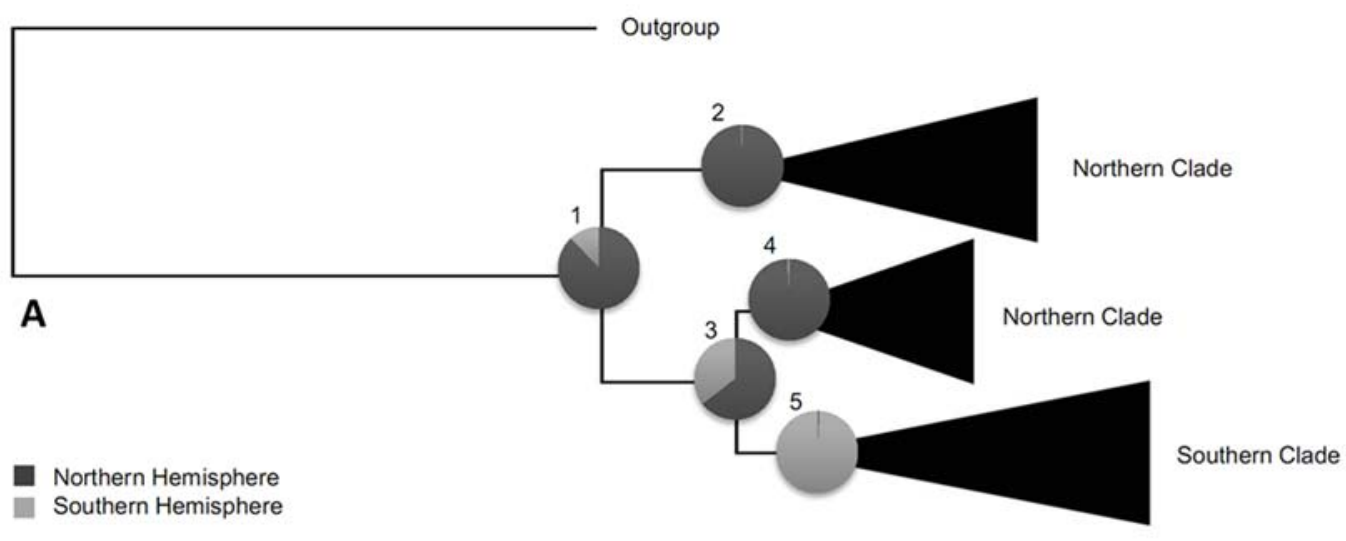

Outgroup

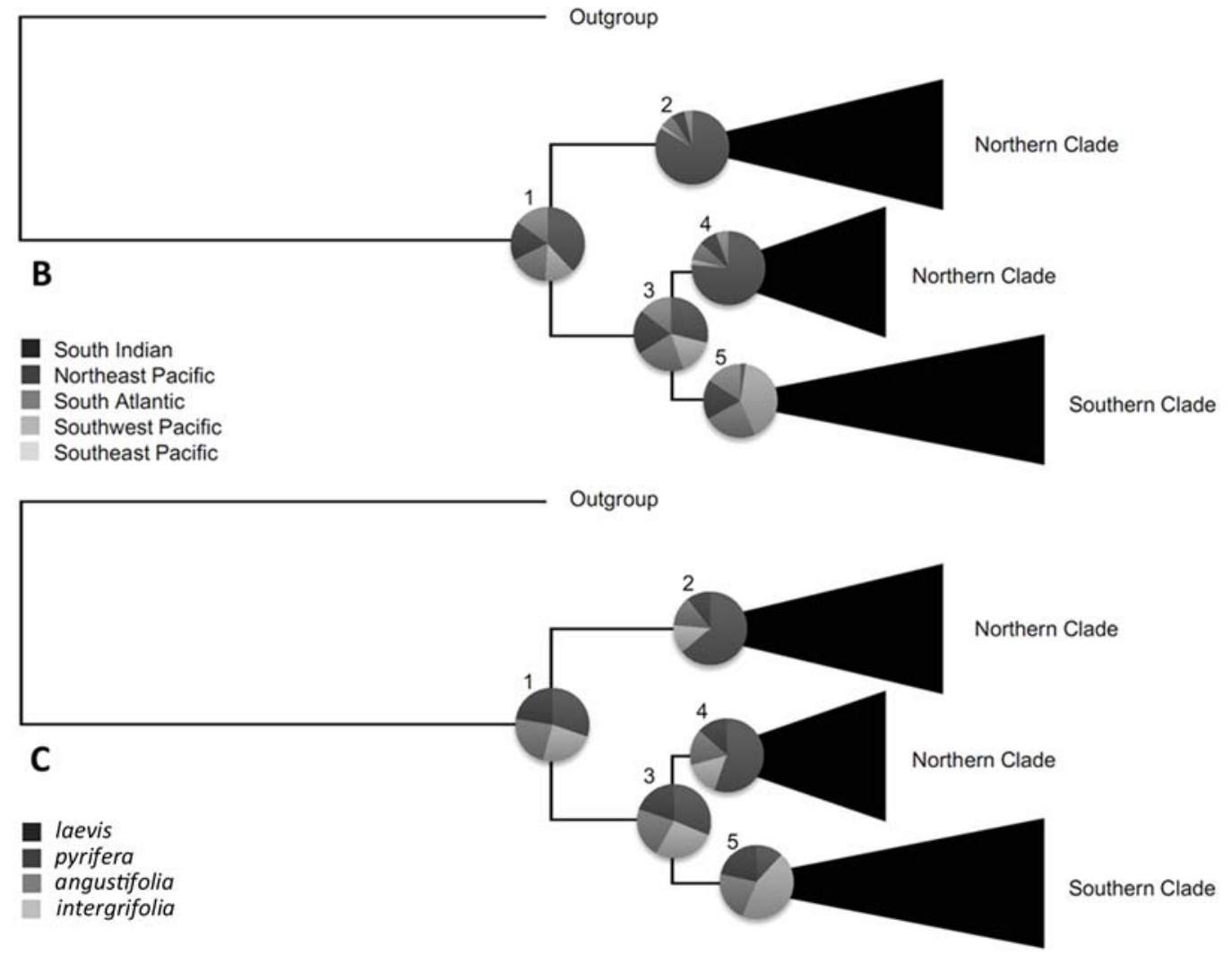

Figure 2. Reconstruction of the ancestral state of the Hemispheric distribution (A), ocean distribution (B), and species level morphology (C), based on both the topology and the branch lengths of the Bayesian consensus tree. The proportion in the pie graphic represents the posterior probability of the ancestral state based on the Bayesian approach / Reconstrucción del estado ancestral de la distribución hemisférica (A), distribución de los océanos (B) y morfología de los morfotipos (C), basado tanto en la topología como el largo de las ramas del árbol de consenso bayesiano. La proporción en el gráfico de torta representa las probabilidades posteriores del estado ancestral 
These comparative phylogenetic analyses sustain that the morphotypes, and its distribution, have a strong phylogenetic signal, indicating that historical process associated to the diversification (i.e., cladogenesis and anagenesis) has determined the actual variability of the morphotypes. These results show that the environmental variation is not the only cause underlying Macrocystis diversity as was previously suggested by Graham et al. (2007) and Demes et al. (2009).

Our results suggest the pyrifera morph is most likely the ancestor of both Northern and Southern Hemisphere clades and would have originated on the Northwestern Pacific coast and later colonized the Southeastern Pacific, ultimately expanding its range throughout most of the Southern Hemisphere. The origin in the Northern Hemisphere would further support the nested pattern of the Southern Hemisphere individuals in Northern Hemisphere clades, as already observed by Coyer et al. (2001). In this study, based on the fact that all clades correspond to a common ancestor, we support the hypothesis of only one biological species assigned to this genus, nominated as Macrocystis pyrifera, just as Demes et al. (2009) previously proposed. They established that the development of distinct Macrocystis holdfast morphologies supports the rejection of the morphological species concept, as well as the collapse of M. pyrifera, $M$. integrifolia and $M$. angustifolia into a single species, with $M$. pyrifera having nomenclatural priority. Within this framework, future studies of the genus Macrocystis should take a population and phylogeographical genetic approach, broadening not only the geographic coverage of the samples, but also the number of individuals sampled in each location, and using more variable genetic markers.

\section{ACKNOWLEDGMENTS}

This study was funded by FONDEF of CONICYT and partially by DID of Universidad Austral de Chile. The authors are grateful for comments and suggestions of anonymous evaluators that greatly improved the manuscript. Jorge Avaria-Llautureo, was supported by CONICYT Master Fellowship.

\section{LITERATURE CITED}

Chin NKM, MT Brown \& MJ Heads. 1991. The biogeography of Lessoniaceae, with special reference to Macrocystis C Agardh (Phaeophyta, Laminariales). Hydrobiologia 215: $1-11$.

Coyer JA, GJ Smith \& RA Andersen. 2001. Evolution of Macrocystis spp. (Phaeophyceae) as determined by ITS1 and ITS2 sequences. Journal of Phycology 37: 574-585.
Dayton PK. 1985. The structure and regulation of some South American kelp communities. Ecological Monographs 55: 447-468.

Demes KW, MH Graham \& TS Suskiewicz. 2009. Phenotypic plasticity reconciles incongruous molecular and morphological taxonomies: the giant kelp, Macrocystis (Laminariales, Phaeophyceae), is a monospecific genus. Journal of Phycology 45: 1266-1269.

Doyle JJ \& JL Doyle. 1987. A rapid DNA isolation procedure for small quantities of fresh leaf tissue. Phytochemical Bulletin 19: 11-15.

Druehl LD, JD Collins, CE Lane \& GW Saunders. 2005. An evaluation of methods used to assess intergeneric hybridization in kelp using pacific Laminariales (Phaeophyceae). Journal of Phycology 41: 250-262.

Estes JA \& PD Steinberg. 1988. Predation, herbivory, and kelp evolution. Paleobiology 14: 19-36.

Graham MH, JA Vasquez \& AH Buschmann. 2007. Global ecology of the giant kelp Macrocystis: from ecotypes to ecosystems. Oceanography and Marine Biology: An annual review 45: 39-88.

Hall TA. 1999. BioEdit: a user-friendly biological sequence alignment editor and analysis program for Windows 95/98/ NT. Nucleic Acids Symposium Series 41: 95-98.

Lane CE, C Mayes, LD Druehl \& GW Saunders. 2006. A Multi-gene molecular investigation of the kelp (Laminariales, Phaeophyceae) supports substantial taxonomic reorganization. Journal of Phycology 42: 493-512.

Lewis PO. 2001. A likelihood approach to estimating phylogeny from discrete morphological character data. Systematic Biology 50: 913-925.

Lewis RJ, M Neushul \& BWW Harger. 1986. Interspecific hybridization of the species of Macrocystis in California. Aquaculture 57: 203-210.

Librado P \& J Rozas. 2009. DnaSP v5: A software for comprehensive analysis of DNA polymorphism data. Bioinformatics 25: 1451-1452.

Lindberg DR. 1991. Marine biotic interchange between the northern and southern hemispheres. Paleobiology 17: 308324.

Lüning K. 1990. Seaweeds. Their environment, biogeography and ecophysiology, 527 pp. John Wiley \& Sons, New York.

Macaya EC \& GC Zuccarello. 2010. DNA barcoding and genetic divergence in the giant kelp Macrocystis (Laminariales). Journal of Phycology 46: 736-742.

Nicholson NL. 1978. Evolution within Macrocystis: Northern and Southern Hemisphere taxa. Proceeding of the International Symposium on Marine Biogeography and Evolution in the Southern Hemisphere 2: 433-441.

Pagel MD \& A Meade. 2004. A phylogenetic mixture model for detecting pattern heterogeneity in gene sequence or character-state data. Systematic Biology 53: 571-581. 
Pagel MD \& A Meade. 2006. Bayesian analysis of correlated evolution of discrete characters by reversible-jump Markov chain Monte Carlo. American Naturalist 167: 808-825.

Pagel MD \& A Meade. 2009. BayesTraits version 1.0 computer package. Software and manual. [on line] < http:// www.evolution.reading.ac.uk/BayesTraits.html>

Pagel MD, A Meade \& D Barker. 2004. Bayesian estimation of ancestral character states on phylogenies. Systematic Biology 53: 673-684.

Parker J, A Rambaut \& OG Pybus. 2008. Correlating viral phenotypes with phylogeny: accounting for phylogenetic uncertainty. Infection Genetics and Evolution 8: 239-246
Westermeier R, D Patiño \& DG Müller. 2007. Sexual compatibility and hybrid formation between the giant kelp species Macrocystis pyrifera and $M$. integrifolia (Laminariales, Phaeophyceae) in Chile. Journal of Applied Phycology 19: 215-221.

Yoon HS, JY Lee, SM Boo \& D Bhattacharya. 2001. Phylogeny of Alariaceae, Laminariaceae, and Lessoniaceae (Phaeophyceae) based on plastid-encoded RuBisCo spacer and nuclear-encoded ITS sequence comparisons. Molecular Phylogenetics and Evolution 21(2): 231-243.

Received 2 February 2012 and accepted 27 August 2012

Associate Editor: Pilar Muñoz M. 